\title{
Remote radioactive seed-loading device for permanent brachytherapy of oral cancer with Au-198 grains
}

\author{
Mukau Sato ${ }^{1}$, Yukari Saito ${ }^{1}$, Toshio Takayama ${ }^{2}$, Toru Omata ${ }^{2}$, Hiroshi Watanabe ${ }^{3}$, Ryoichi Yoshimura ${ }^{4}$ \\ and Masahiko Miura ${ }^{5}$
}

\begin{abstract}
This paper presents a remote radioactive seed-loading device for permanent brachytherapy of oral cancer with Au-198 grains to reduce the exposure dose to the doctors. Permanent brachytherapy is a treatment which involve implanting of radioactive Au-198 grains, and requires neither long-term needle insertion nor diet-restriction. This is its advantage over temporary brachytherapy. However, the doctors that perform this therapy are exposed to radiation during the treatment procedures. This hinders permanent brachytherapy from being commonly used. The developed device shoots an Au grain from a distant position using air pressure and loads it onto the applicator. This can reduce the exposure dose of the doctors compared with that of the traditional procedure because the doctors are able to stay as far away from the radioactive seeds as possible. We conducted experiments of motion simulation of the proposed and traditional treatment procedures, and compared the exposed doses. The device can reduce the exposed dose by $62 \%$ on average.
\end{abstract}

Keywords: Brachytherapy, Oral cancer, Loading device

\section{Introduction}

In recent years, radiotherapy has attracted attention as a treatment for oral cancer because it can maintain a better quality of life (QOL) of the patient in comparison with surgery. Among the radiotherapies, brachytherapy has a number of advantages; it can intensively irradiate cancer tissue and reduce radiation exposure to normal tissue around the cancer tissue. With regard to oral cancer, two brachytherapy methods are performed in Japan. One is a temporary method using Ir-192 or Cs-137 needles, and the other is a permanent method using $\mathrm{Au}-198$ grains $[1,2]$. The latter requires implanting small radioactive $\mathrm{Au}$ grains $(2.5 \mathrm{~mm}$ in length and $0.8 \mathrm{~mm}$ in diameter), and involves no diet-restriction, whereas the former requires a 3-5 day diet-restriction period during which needles are inserted intra-orally to the cancerous

\footnotetext{
*Correspondence: takayama@pms.titech.ac.jp

${ }^{2}$ School of Engineering, Tokyo Institute of Technology, 4259-G5-27 Nagatsuta-cho, Midori-ku, Yokohama, Kanagawa 226-8502, Japan Full list of author information is available at the end of the article
}

tissue. Therefore, the permanent method can be applied to aged or handicapped patients for whom the temporary method is not suitable. Moreover, it can be applied to oral cancers other than carcinoma of the tongue, such as carcinomas of the floor of the mouth and the buccal mucosa, and oropharyngeal cancer [3].

The doctors are exposed to radiation during the treatment procedures. Consequently, there are few hospitals conducting permanent brachytherapy, and the number of treatments is limited at these hospitals. Brachytherapy devices for prostate and lung cancers that reduce the radiation dose to the doctors have been developed [49]. However, devices that can be applied to permanent brachytherapy for oral cancer have not been developed.

As mentioned next, oral cancer is quite different from prostate and lung cancers including their surrounding environments. Therefore, brachytherapy methods for them are also different and devices developed for prostate and lung cancers are not applicable for oral cancers.

Therefore, the purpose of this paper is to develop such a device to make permanent brachytherapy standard in 
Japan and worldwide. In south and south-east Asian countries, such as India where chewing-tobacco is popular, it is said that $30 \%$ of cancers are oral cancers. Therefore, there is a potential demand for the device developed in this paper.

In the treatment, the doctor implants 8-15 Au grains in and around the cancer tissue with the applicator shown in Fig. 1. The doctor is exposed to radiation during the preparation process (Fig. 2a) in which the Au grains are loaded into the applicator, and during the operating process (Fig. 2b) in which the Au grains are implanted in the patient. In the preparation process, the doctor loads $\mathrm{Au}$ grains one by one with a tweezer into applicators arranged on a tray. In this process, the doctor needs to stand close to the radioactive seeds for a long time. In the operating process, the doctor is exposed to radiation from applicators arranged on a tray, holding the applicator to implant an Au grain, and grains already implanted in the patient.

The differences between oral cancer and prostate or lung cancer lead to differences in the design of devices, which can reduce exposure dosage. The permanent brachytherapy treatment for prostate cancer uses approximately 15-20 applicators, wherein each applicator is loaded linearly with radioactive seeds and spacers. Each applicator implants them linearly at a time to surround the cancer tissue. To reduce the exposure dose of the doctors, implantation robots were developed [4-7]. They use an ultrasonic probe, CT, or MRI to implant the grains accurately, as prostate cancer cannot be observed directly via visual inspection. A device that automates the loading process of the seeds and spacers into the applicators was also developed. The device developed by Green [8] arranges the seeds and spaces on the ditches of cartridges and slides them into a needle using stylets. In the brachytherapy treatment for lung cancer, linearly arranged
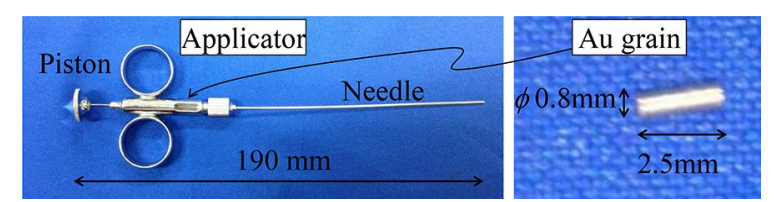

Fig. 1 Applicator

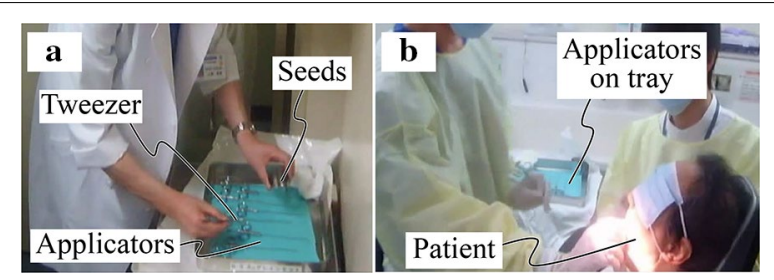

Fig. 2 Scenes from traditional brachytherapy. a Applicators arranged on a tray. $\mathbf{b}$ Scene of the implantation process seeds and spacers are implanted in a manner similar to the method observed in brachytherapy for prostate cancer [9].

Unlike prostate cancers, oral cancers occur on the surface or close to the surface of the oral organs. Au grains need to be implanted one by one. Therefore, the devices developed for prostate or lung cancer cannot be used for the brachytherapy of oral cancer. For temporary brachytherapy, Remote After Loading System (RALS) is commercially available [10]. However, it cannot be used for the permanent brachytherapy of oral cancer.

In our previous study, we developed a remote needle insertion manipulator for the permanent brachytherapy of oral cancer [11]. The manipulator can generate pivot motion by a Remote Center-of-Motion Mechanism (RCM) so that it can insert a needle into the narrow oral space. The whole manipulator system becomes large and complicated resulting in a high-class medical device according to the medical law in Japan. This makes its early introduction to clinical practice difficult.

In this study, we develop a remote radioactive seed-loading device. It shoots an Au grain using air pressure from a large distance and loads it into a traditional applicator, as shown in Fig. 3. It can reduce exposure dose by minimizing the time for which the doctor is close to the radioactive seeds. The device itself does not make contact with the patient, which is advantageous for early introduction to clinical practice. It can also be used to load seeds into the remote needle insertion manipulator, as mentioned.

This is because the manipulator system uses the same applicator. The developed seed loading device can also automate the seed loading process for the manipulator system. Otherwise, manual loading is necessary, which would increase the exposure dose of the doctor.

Therefore, to develop the remote loading device prior to the remote needle insertion manipulator is a reasonable choice.

"Remote loading device" section discusses the design of the remote radioactive seed-loading device and describes its components. "Experiment" section shows the experiment of simulation motions by subjects to evaluate the exposure dose in the traditional and proposed brachytherapy procedures. The effectiveness of the exposure dose reduction is confirmed. "Discussion" section discusses the effectiveness of the proposed device and why commercially available devices, such as parts feeder systems and pneumatic tube conveyors cannot be employed for this remote seed-loading device.

\section{Remote loading device Proposed system}

The remote loading device is composed of a delivering unit, an applicator holder, and a pipeline connecting 

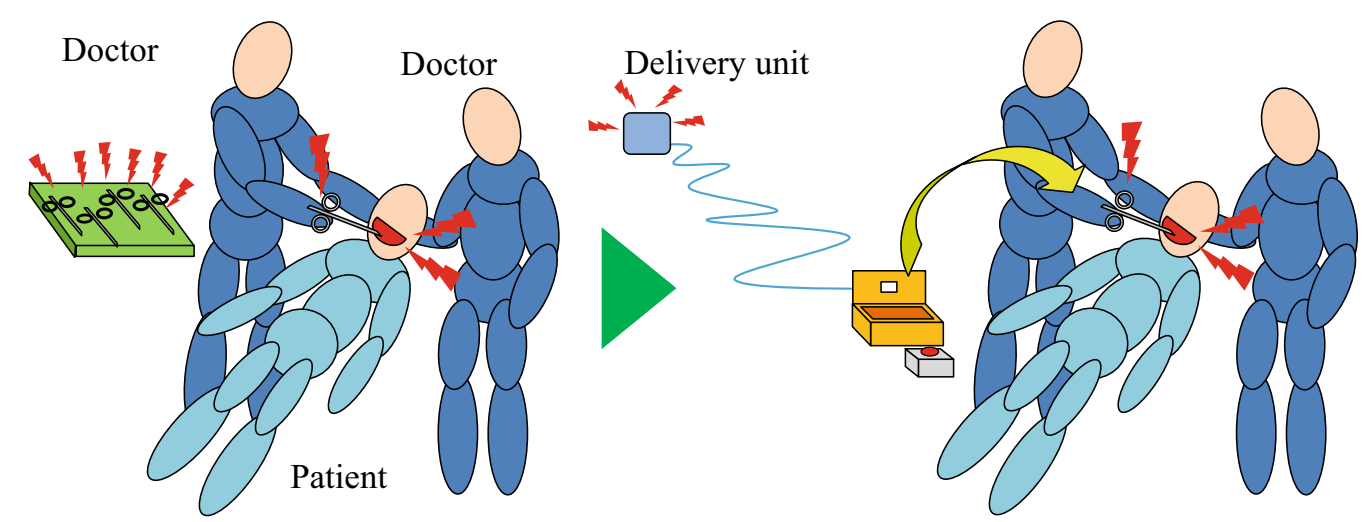

Fig. 3 Traditional and proposed brachytherapy procedures

them, as shown in Fig. 4. The delivering unit shoots the arranged seeds one by one. The applicator holder catches the shot seed to load it into the applicator. The pipeline transmits the seed, and is typically $10 \mathrm{~m}$ long.

The proposed procedure using the device is as follows:

1. First, the doctor puts seeds into the aligning subdevice, as shown in Fig. 5, and inserts it into a vibrator that aligns the seeds by vibration. During the seed alignment, the doctor can stay away from the seeds and exposure dose can be reduced.

2. The doctor sets the aligning sub-device to the delivering unit. The delivering unit is set far from the dispensary; for example, it can be set in the next room. Consequently, the exposure dose from the applicators placed on the tray in the traditional method can be eliminated.

3. In the treatment to the patient, the doctor sets an applicator into the holder and switches to trigger seed sending. Then, a seed is shot and loaded into the

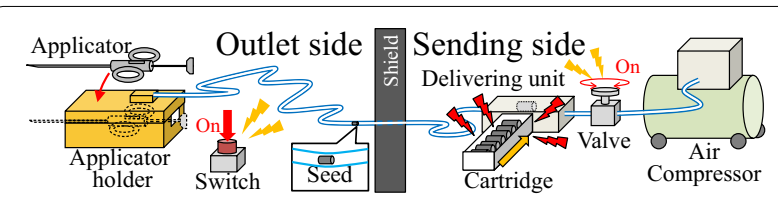

Fig. 4 Concept of the remote radioactive seed-loading device

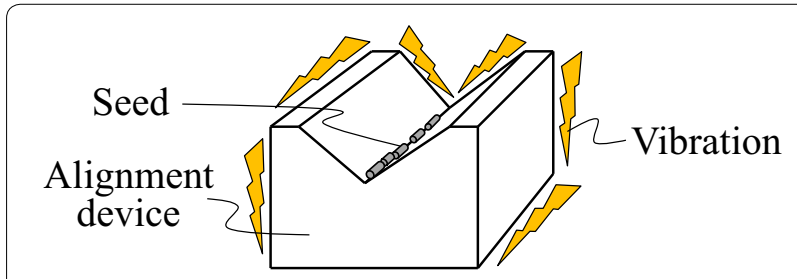

Fig. 5 Aligning sub-device applicator passing through the pipeline. The doctor implants the seed and repeats this process until all seeds are implanted.

Figure 6 shows the developed remote seed-loading device, which satisfies the following design requirements. When a novel device is introduced to the clinical practice, the change in clinical environment or manner increases the fatigue of the doctor and, therefore, this should be avoided. A novel device should also be easy to operate for the doctor. Therefore, it should not require expertise and complex techniques to use. Moreover, to send a seed from far away, it needs to be shot by air pressure. To prevent back-flow, sealing is necessary. If sealing materials make sliding motions, there is possibility that small powder dusts will adhere to the seed. Therefore, dust generation must be avoided. The next subsections describe details of the developed device.

\section{Components of the device Seed-aligning sub-device}

Figure 7 shows the V-shaped aligning sub-device. We conducted experiments to verify that seeds can be aligned in the bottom groove of the $\mathrm{V}$-shape with a vibrator and measure the time needed to align them. Throughout this paper, we use mock seeds made of tungsten with

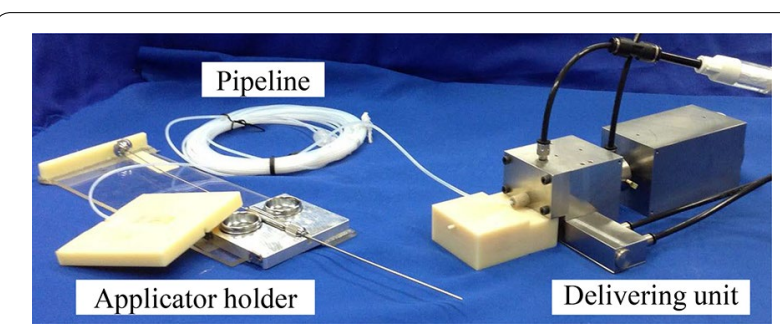

Fig. 6 Overview of the remote radioactive seed-loading device 


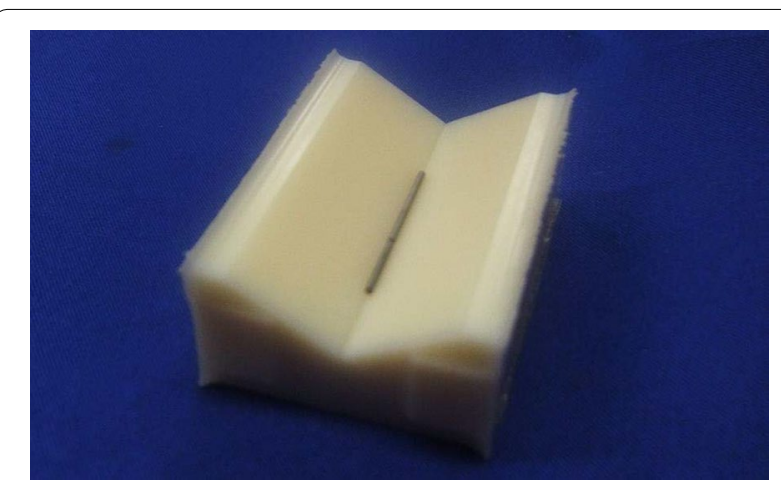

Fig. 7 Developed V-shaped aligning sub-device

the same dimensions as the Au grain. The density of tungsten is close to that of gold. The measurement was repeated 5 times. Figure 8 shows the result. The horizontal axis shows the number of seeds to align and the vertical axis the time needed to align them. All seeds can be aligned, although the time needed increased as the number of seeds increased.

\section{Seed-setting sub-device}

Figure 9 shows the overview of the delivering unit and Fig. 10 its cross sections: the front view (left) and side

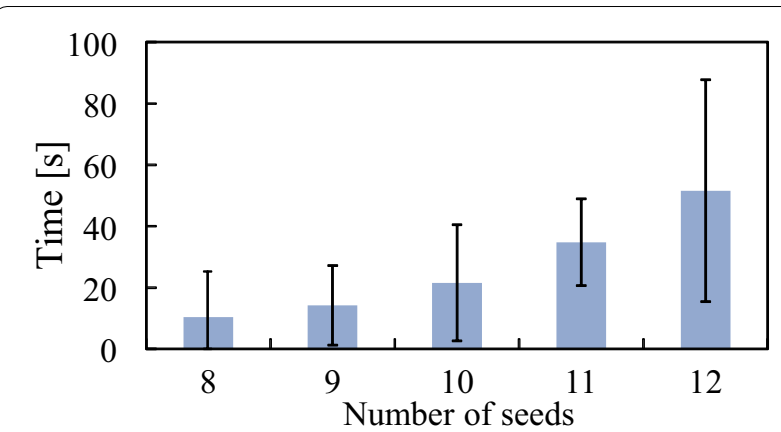

Fig. 8 Relationship between the number of seeds and time needed to align

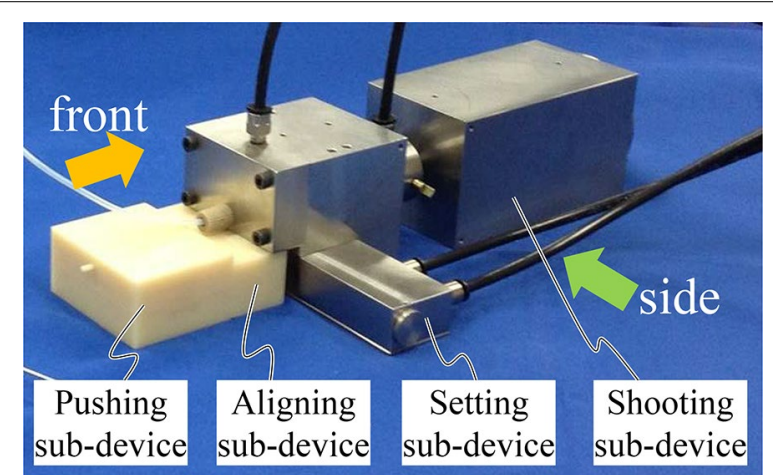

Fig. 9 Overview of the delivering unit

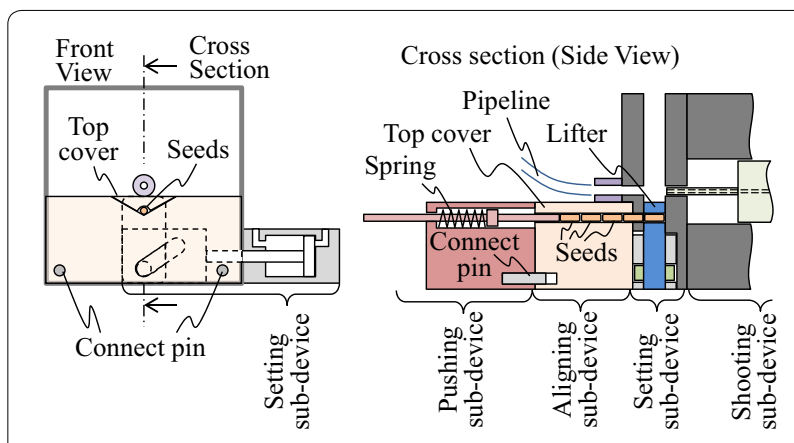

Fig. 10 Cross sections of the delivering unit front view (left) and side view (right) showing the connection of four sub-devices

view (right). From the front of the unit, four sub-devices are arranged. Those are the seed pushing, aligning, setting, and shooting sub-devices. The seed pushing subdevice has a pushing rod pushed by a spring, and it is connected to the seed-aligning sub-device with two pins. The pushing rod pushes the aligned seeds into the seed-setting sub-device with the spring. The head seed is entered into the hole of the lifter in the seed-setting sub-device.

Figure 11 shows the mechanism of the seed-setting sub-device. As shown in Fig. 11b, air pressure is supplied to its cylinder and a cam mechanism of the piston head lifts up the lifter. The lifter carries the head seed to the route of the pipeline one by one. The seed-shooting subdevice driven by air pressure carries the seed into the pipeline and shoots it, as shown in Fig. 11c. Finally, the seed-shooting sub-device moves backward; the seed-setting device moves down its lifter, and the next seed is set in the lifter, as shown in Fig. 11d.

The linearly arranged seeds may cause a jam when a seed is lifted up to the route of the pipeline. To avoid such a jam, a taper is cut in the lifter, as shown in Fig. 12. The length of the Au grain is $2.50 \pm 0.01 \mathrm{~mm}$, and the width of the lifter is set to be $2.6 \mathrm{~mm}$. Thus, in addition to the head seed, the front of the next seed is entered into the hole of the lifter. When the lifter moves upward, the surface of the taper collides with the front end of the next seed, which generates a friction force at the tapered surface. If the angle of the taper is sufficiently large (for example, $70^{\circ}$ ), the lifter can overcome the friction and push the next seed back by the taper and therefore the jam can be avoided (Fig. 12b).

\section{Seed-shooting sub-device}

Figure 13 shows the motion of the seed-shooting subdevice. When solenoid valve 1 is activated (Fig. 13b), the pressured metal bellows push forward the piston. The piston has a thin pipe that pushes the seed to a prescribed position. At the same time, a small projection from the 

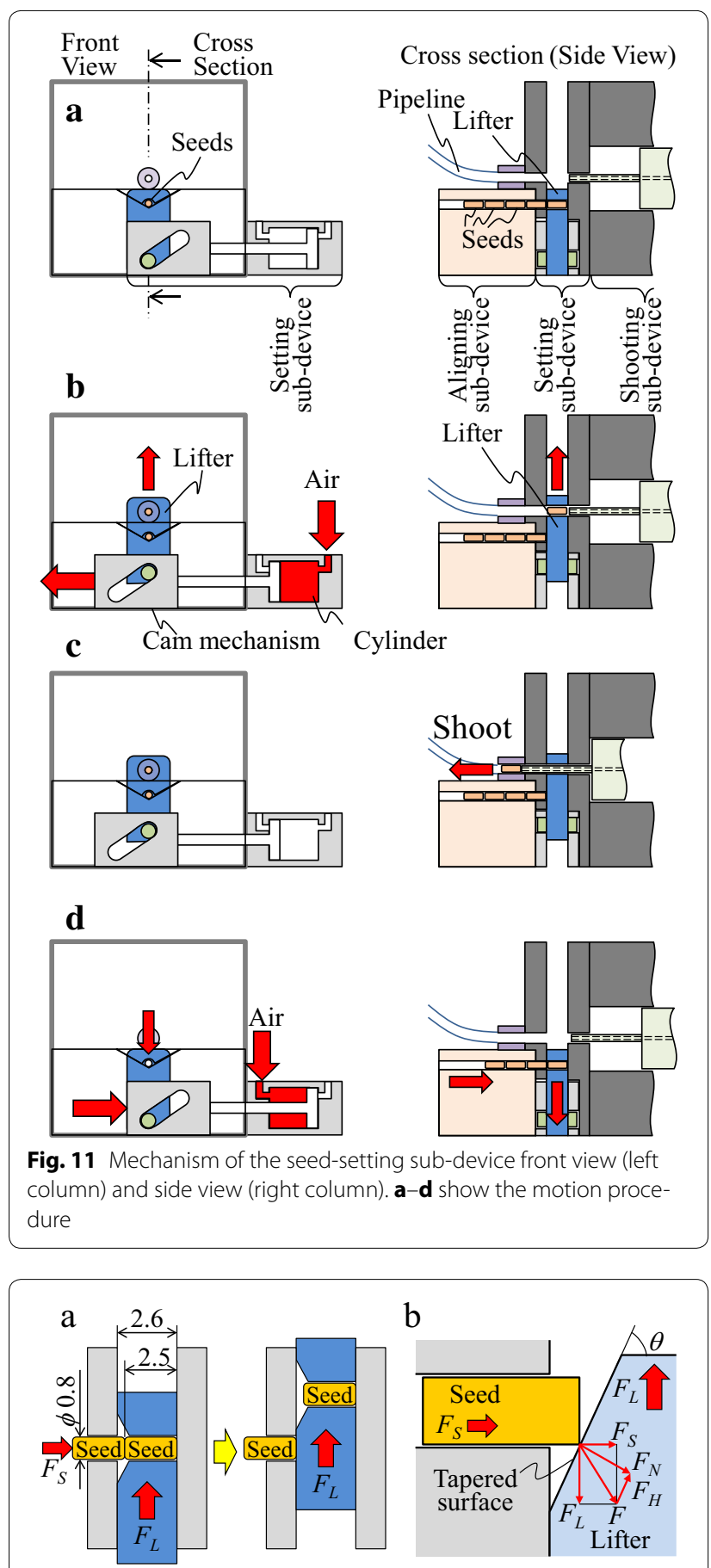

Fig. 12 Measure to prevent seed jamming

piston pushes a switch attached to the seed-setting subdevice, which activates solenoid valve 2 and supplies air to shoot the seed (Fig. 13c).

Figure 14a shows the metal bellows used to generate the reciprocation motion of the pipe. It is structurally simple and has some advantages, such as its high airtightness, its ability to extend by air pressure without
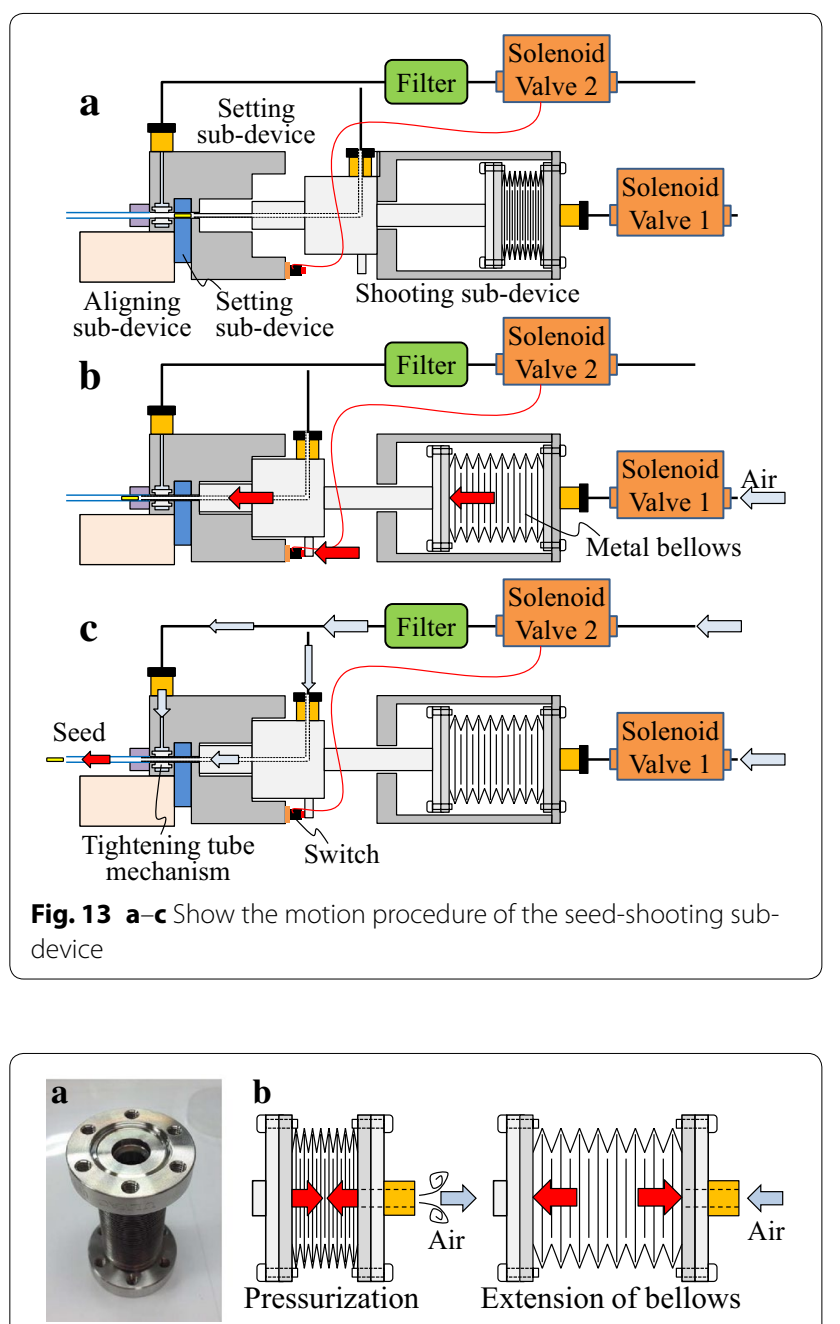

Fig. 14 Metal bellows. a Photo. b Cross section and its motion

friction, and its ability to spring back by its resilience (Fig. 14b). Therefore, an additional spring to return it to the initial position is not required.

In the motion procedure in Fig. 13, a tube tightening mechanism is also activated. Its conceptual diagram is shown in Fig. 14. It tightens the front tip of the pipe to prevent backward air leak when the seed is shot. Usually, O-rings are used to prevent air leak from sliding portions. However, O-rings generate abrasion and some dusts could attach to the seed. Therefore, to avoid the generation of dusts, we propose the tightening tube mechanism. It is composed of a silicone rubber tube with cylindrical spacers in its inside, and a fixture with an air channel that provides air pressure to the silicone rubber tube from the outside. First, the pipe pushes the seed into the pipeline at a prescribed position (Fig. 15a-1). Next, the air chamber is pressurized and the silicone rubber tube is deformed from its outside (Fig. 15b-2), which prevents 


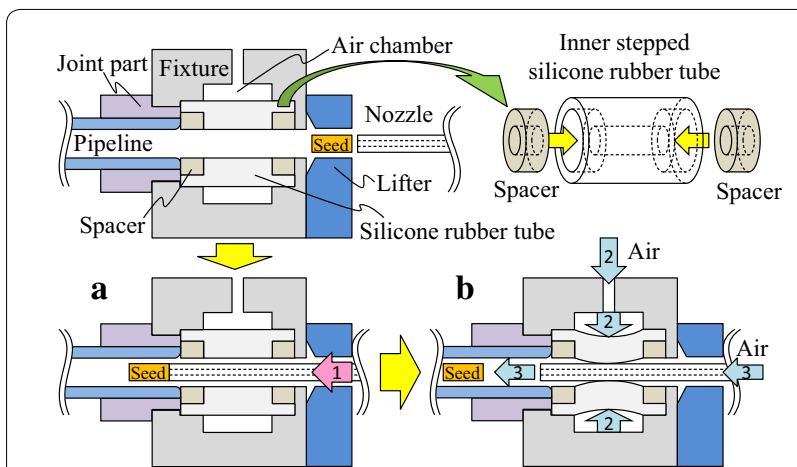

Fig. 15 Tube tightening mechanism. a-b Show the motion procedure

backward air leak when the nozzle of the pipe blows air to shoot the seed (Fig. 15b-3). It does not rub against the nozzle while the nozzle pushes the seed into the pipeline. Only when it is activated does it tightens up the pipe and prevents the backward air leak.

The air that shoots seeds also requires the factor of cleanliness. To ensure clean air, we use air filters used for medical and food automation devices. Moreover, we use solenoid valves for a clean room.

\section{Applicator holder}

The applicator holder that loads a seed into an applicator has a box-like shape and the pipeline is connected to its lid (Fig. 16). The shot seed falls into the loading hole of the applicator (Fig. 17a), and by pushing the piston by
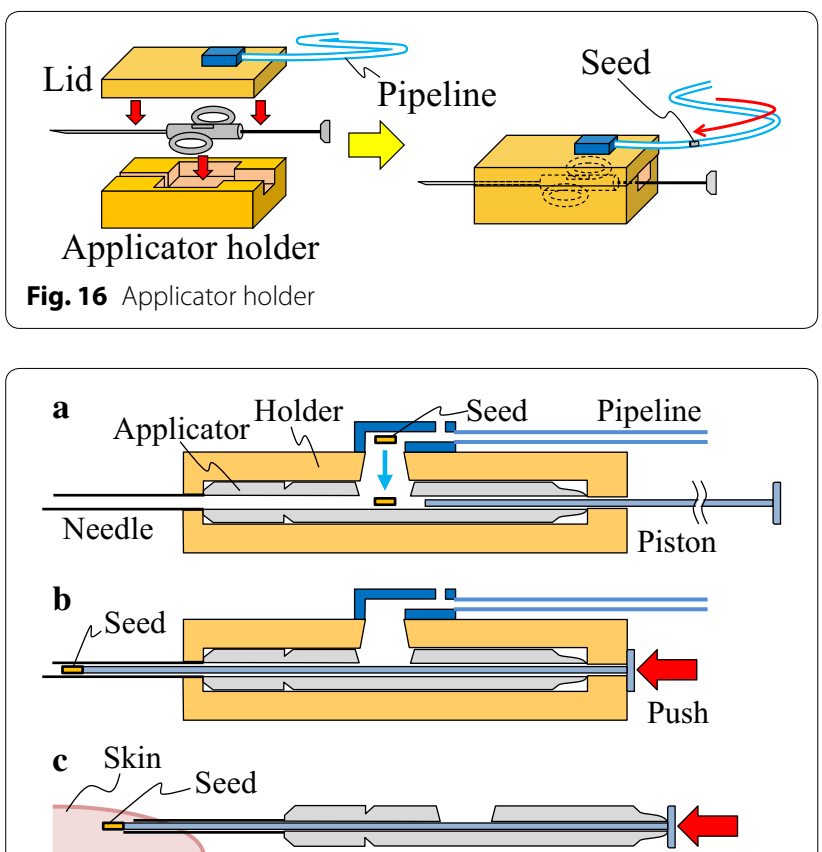

Fig. 17 Mechanism of the applicator holder. a-c Show the motion procedure half of its length, the seed is loaded into the needle of the applicator (Fig. 17b). When the needle is inserted into the skin and the piston is pushed completely, the seed is implanted under the skin (Fig. 17c).

\section{Experiment}

\section{Operation verification}

We developed the remote loading device out of stainless steel and verified its operation as follows:

1. The piston of the seed-setting sub-device can generate reciprocation motion so that its cam mechanism works with an air pressure of $0.25 \mathrm{MPa}$, as shown in Fig. 18.

2. The hole of the lifter of the seed-setting sub-device is positioned accurately with respect to the pipe of the seed-shooting device by penetrating them with a needle.

3. The seed-shooting sub-device can push a seed into the pipeline and can shoot it. It passes through a pipeline of $10 \mathrm{~m}$ in less than $2 \mathrm{~s}$.

Next, we connected the seed aligning, setting, and shooting sub-devices, to confirm that the sub-devices can work together continuously. The lifter of the seed-setting subdevice lifted up a seed to the route of the pipeline, and the nozzle of the shooting sub-device pushed the seed into the pipeline, and the seed was shot. This test succeeded continuously more than 100 times.

\section{Evaluation experiment of the device}

To estimate the exposure dose, using real radioactive $\mathrm{Au}$ grains should be avoided. Instead, we estimate it by measuring the time and distance from the mock seeds made of tungsten in motion simulations performed by participants. It can be estimated by

$$
E=\int f \frac{1}{D^{2}} d t
$$

where $\mathrm{E}[\mu \mathrm{Sv}]$ is the exposure dose, $f\left[\mu \mathrm{Sv} \mathrm{m}^{2} / \mathrm{s}\right]$ is the radioactive dose coefficient, and $D(t)[\mathrm{m}]$ is the distance between a radioactive seed and a reference point on the participant. It is on the left chest where the doctor wears a dosimeter in the traditional brachytherapy treatment.

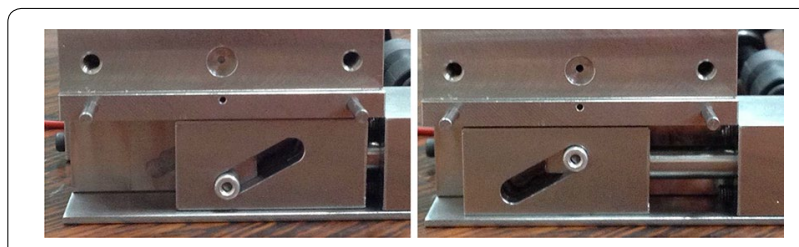

Fig. 18 Test of the seed-setting sub-device 
The radioactive dose coefficient $\mathrm{f}$ is defined as a coefficient for dose evaluation from external exposure, and it can be calculated by the product of the effective dose ratio constant and nominal radioactivity value. From the physical characteristics of the gold grain, they are 0.0576 $\mu \mathrm{Sv} \mathrm{m} /(\mathrm{MBq} \mathrm{h})$ and $185 \mathrm{MBq}$, respectively. Thus,

$$
f=0.0576 \times 185 / 3600=2.96 \times 10^{-3} \mu \mathrm{Sv} \mathrm{m}^{2} / \mathrm{s}
$$

The participants separately performed motion simulations for the three processes: (1) the seed-aligning process, (2) the seed-loading process, and (3) the traditional seed-loading process. These are described next. We recorded with a stereo camera to measure the time and distance. The attached software can calculate the distance between two arbitrary points on the basis of triangulation. We obtained the distance at every second from the pictures sampled from the movie.

This experiment was approved by the Ethical Committee of Tokyo Institute of Technology, and advertised for the participants of the experiment. Written informed consent was obtained from each participant. The number of the participants is five. They are men and women in their 20s. The participants practice two to five times to become skillful before recording the experiment. The number of seeds is 8 . The participants wore a fatigue uniform (Fig. 19) that has a mark at the left chest, which helps recognize the position of the left chest in the picture.

\section{Motion simulations}

1. Motion simulation of the seed-aligning process

a. Carry a case containing seeds from a pre-positioned location (about $3 \mathrm{~s}$ ).

b. Throw the seeds into the aligning sub-device, set it in a vibrator, and turn it on (Fig. 20a).

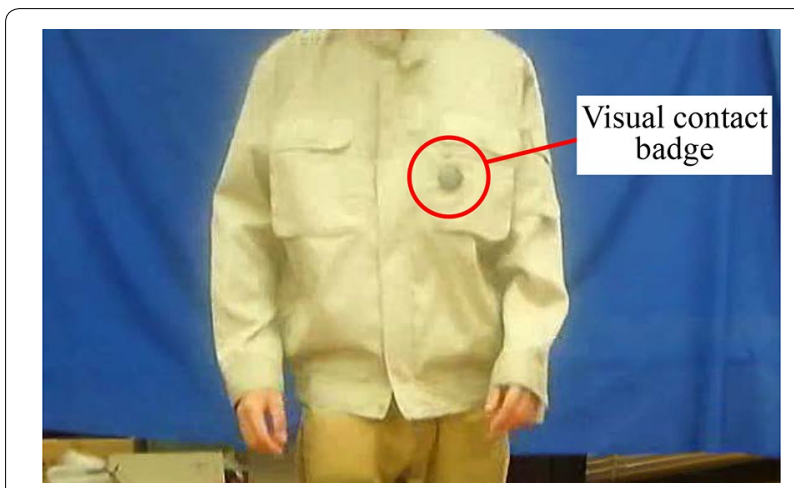

Fig. 19 Uniform for the participant

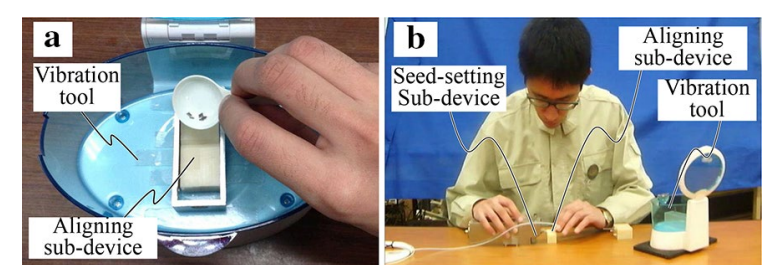

Fig. 20 a Aligning sub-device in the vibration tool. b Scenes in the motion simulation of the seed-aligning process

c. Keep at least $1 \mathrm{~m}$ away from the vibrator, and wait until the seeds are aligned automatically (about $40 \mathrm{~s}$ ).

d. Turn off the vibrator and put the top cover on the seed-aligning sub-device, and set it to the seed-setting sub-device (Fig. 20b).

The obtained exposure dose is denoted as $P A$ in Table 2.

2. Motion simulation of the seed-loading process

a. Set an applicator in the applicator holder and switch it on to trigger the seed shooting.

b. Open the lid of the applicator holder and confirm that the seed falls in the loading hole of the applicator. Then push the piston of the applicator to load the seed into its needle.

In actual treatment, seed implantation to the patient would follow B. The participant repeats processes A and $\mathrm{B} \mathrm{n}$ times, where $\mathrm{n}$ is the number of seeds.

Meanwhile, the doctor is exposed to radiation from the seeds implanted in the patient. We estimate the exposure dose of implanted seeds during the seedloading process by using the distance and time measured from a video on the traditional actual treatment. Figure 21 shows the outline figures of the applicator exchange and the needle insertion in the traditional implanting process, while Table 1 shows the data of the distance and time. Based on the distance between the doctor and the target tumor, we estimate the exposure dose radiated from the tumor. The obtained exposure dose is denoted as $P L$ in Table 2.

We estimate the exposure dose during the seed implantation to the patient from Table 1 . The exposure dose in the proposed method is only from the holding applicator and the already implanted seeds around the target tumor because no applicators are put on a tray.

We applied these data to eq. (1) and obtained the exposure dose, which is denoted as $P H$ in Table 2. Therefore, the total exposure during process $P I$ is calculated by $P I=P L+P H$. 
The total exposure dose of the proposed method is the sum of those of the seed-aligning process and the implanting process.

3. Motion simulation of the traditional seed-loading process

a. Carry a case containing seeds from a pre-positioned location (about $3 \mathrm{~s}$ ).

b. First, push the piston into the applicator completely. Using tweezers, drop the first seed into the loading hole of the applicator, as shown in Fig. 22a. For the rest of the seeds, put them at the indicated places on the paper, as shown in Fig. 22b (this is because we have only one applicator).

c. Pick up the applicator and pull its piston to drop the seed into the bottom of the loading hole. Then, push the piston again. This motion is repeated $n$ times.

d. Carry the applicator to a hanger (about $10 \mathrm{~s}$ ).

The exposure dose dureing traditional seed-loading process is denoted as $T L$ in Table 2.

Moreover, we calculated the exposure dose during the traditional implantation process from the data of Table 1 .
In this calculation, the radiation from the applicators on the tray is added. The obtained data is denoted as $T I$ in Table 2.

The total exposure dose of the traditional method is the sum of those of the traditional seed-loading process and the implantation process. The proposed remote loading device can reduce the exposure dose by $44-68 \%$ and by $62 \%$ on average. The heights, postures, and progress degrees of the participants are the factors of this scattering.

\section{Discussion \\ Effectivenes}

The acceptable radiation dose per year is recommended by the International Commission on Radiological Protection (ICRP). ICRP recommends $100 \mathrm{mSv} / 5$ year (with a maximum of $50 \mathrm{mSv} /$ year). This restriction limits the number of treatments. If the $62 \%$ of exposure dose can be reduced, doctors engaging in oral brachytherapy can increase the number of treatment by $2.6(=1 /(1-0.62))$. This is the contribution of the proposed device.

Possibility to employ commercially available devices: Parts feeder systems are commercially available [12]. They can feed parts when a large number of similar parts are supplied in its resource area. They direct parts initially at random orientations to a feed track and sends out the aligned parts one by one. In this process, parts

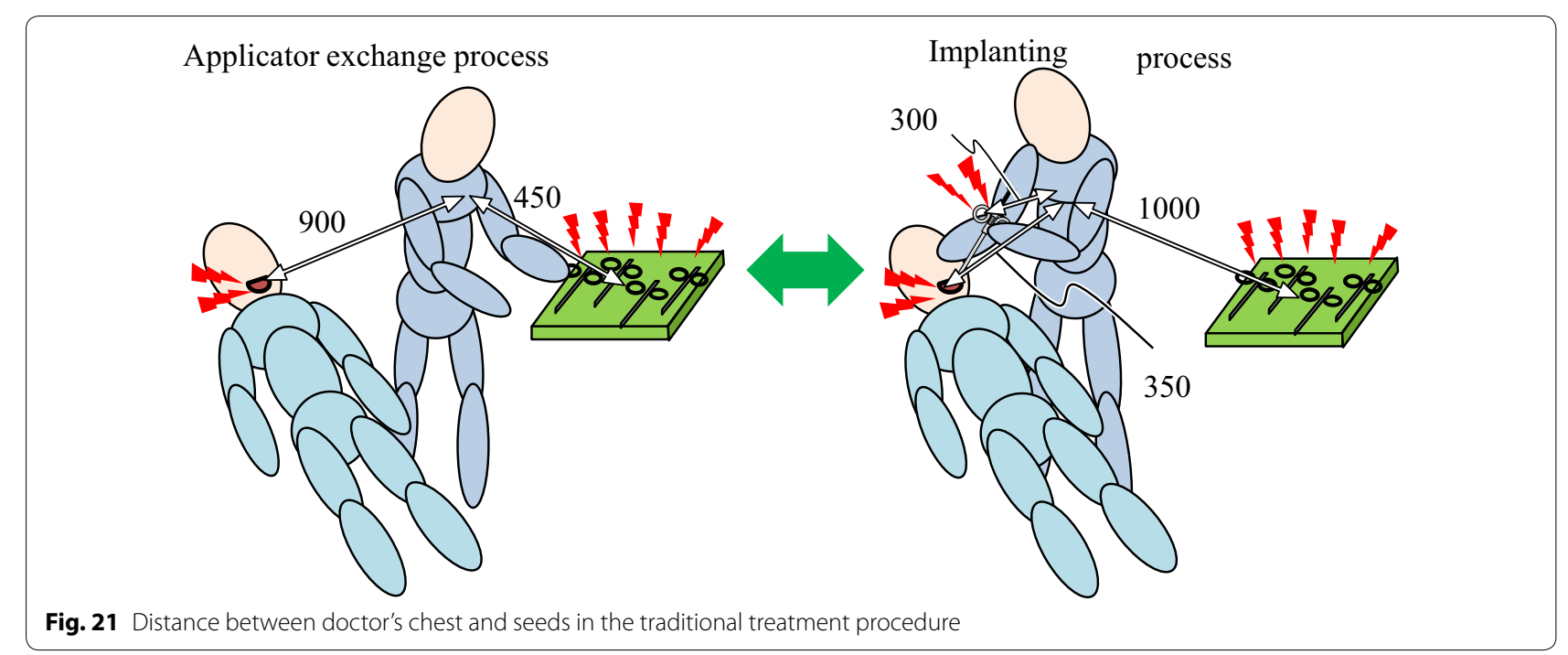

Table 1 Time and distance between doctor's chest and seeds in the traditional treatment procedure: distance $D(\mathrm{~mm})$, time $t(s)$

\begin{tabular}{|c|c|c|c|c|c|c|}
\hline & \multicolumn{2}{|c|}{ Applicators on tray } & \multicolumn{2}{|c|}{ Holding applicator } & \multicolumn{2}{|c|}{ Target tumor } \\
\hline & $D$ & $t$ & $D$ & $t$ & $D$ & $t$ \\
\hline Changing & 450 & 5 & - & - & 900 & 5 \\
\hline Implanting & 1000 & 15 & 300 & 15 & 350 & 15 \\
\hline
\end{tabular}


Table 2 Experimental results

\begin{tabular}{|c|c|c|c|c|c|}
\hline Subject & a & b & C & d & e \\
\hline$P A(\mu S v)$ & 10.2 & 17.7 & 6.51 & 17.2 & 16 \\
\hline$P L(\mu S v)$ & 5.02 & 4.52 & 2.99 & 2.78 & 6.5 \\
\hline$P H(\mu S v)$ & 5.48 & & & & \\
\hline$P I(\mu S v)$ & 10.5 & 10.0 & 8.47 & 8.46 & 12 \\
\hline Total $($ proposed $)(\mu S v)=P A+P I$ & 20.7 & 27.7 & 15.0 & 25.6 & 28 \\
\hline$T L(\mu S v)$ & 40.3 & 63.0 & 17.4 & 39.7 & 24 \\
\hline$T I(\mu S v)$ & 26.0 & & & & \\
\hline Total (traditional) $(\mu \mathrm{S} v)=\mathrm{TL}+\mathrm{TI}$ & 66.3 & 89.0 & 43.4 & 65.7 & 50 \\
\hline Reduction ratio (\%) & 68.8 & 68.9 & 65.4 & 60.9 & 44 \\
\hline
\end{tabular}

$P A$ exposure dose during the seed-aligning process

$P L$ exposure dose from the applicator in which a seed is loaded and already implanted seeds in the patient during the implantation process

$\mathrm{PH}$ exposure dose during the seed implantation to the patient which is estimated from the traditional process

$\mathrm{Pl}$ exposure dose during the implantation process $(\mathrm{PL}+\mathrm{PH})$

$T L$ exposure dose during the traditional seed-loading process

$\mathrm{Tl}$ exposure dose from the handling applicator, implanted seeds and prepared applicators beside the doctor during the traditional implantation process

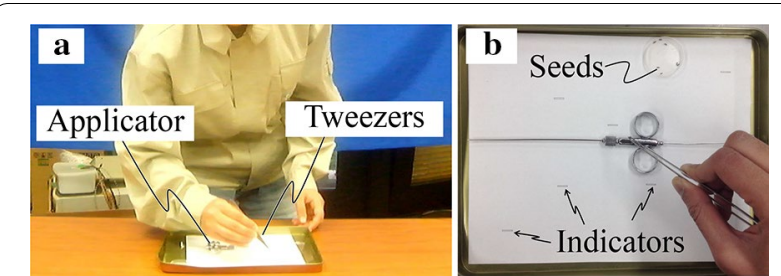

Fig. 22 a Scene in motion simulation of the traditional seed-loading process. $\mathbf{b}$ Top view of the applicator

that are at undesirable orientations are dropped into the resource area and sent back to the track.

Therefore, conventional parts feeders have to prepare more parts than the ones required.

$\mathrm{Au}$ grains that are used for the brachytherapy treatment of oral cancer are radioactive materials, and a large number of grains cannot be provided because the radiation dose and cost increase.

A few special parts feeder systems can send out each and every part. Such parts feeder systems may be used instead of the vibrator used in this study. However, they align the parts by repeating the trial process aforementioned, and hence, the time required to send out all the $\mathrm{Au}$ grains is not guaranteed. In addition, the number of radioactive $\mathrm{Au}$ grains must be controlled. Before the treatment, the number of aligned $\mathrm{Au}$ grains should be confirmed via visual observation.

Therefore, Au grains need to be aligned and saved in a buffer prior to the treatment even if such commercial parts feeder systems are used. A supplying device that sends the aligned Au grains in the buffer into a pneumatic tube conveyor one by one is necessary during the treatment. The pushing subdevice and the setting subdevice that we developed serve as a supplying device, and therefore, they are essential.

Pneumatic tube conveyors that shoot parts supplied by parts feeder systems are slso commercially available [13]. However, they are designed to shoot specific parts, e.g., bolts or nuts. Air leak in a pneumatic tube conveyor may be negligible for sufficiently heavy parts such as bolts and nuts. Au grains are small and lightweight, and an air leak can blow away subsequent grains in the buffer.

Moreover, in the commercial pneumatic tube conveyor combined with a parts feedersystem, parts are supplied via the pushing force of a parts feeder system. However, as mentioned, Au grains must be aligned before the treatment, and cannot be supplied by the pushing force of a part feeder system during the treatment. Therefore, a special pneumatic tube conveyor needs to be designed to shoot Au grains. The shooting subdevice that we developed satisfies the requirement for shooting Au grains.

\section{Conclusion and future work}

To reduce the exposure dose to the doctor in the permanent brachytherapy of oral cancer, we developed a remote radioactive seed-loading device that delivers radioactive seeds from a distance to the doctor using air pressure to load the seed into the applicator. To confirm the effectiveness of the device, we conducted motion simulations of the proposed and traditional brachytherapy procedures and compared their exposure doses. The results show that the proposed procedure using the developed device can reduce the exposure dose by $62 \%$ on average. In future, we will apply the proposed procedure for practical purposes.

\section{Authors' contributions}

MS, YS, TT and TO equally contribute to the development of the device and conducting the experiments. HW, RY and MM equally contribute to the overall system design from the medical point of veiw. All authors read and approved the final manuscript.

\section{Author details}

1 Department of Mechano-Micro Engineering, Tokyo Institute of Technology, 4259-G5-27 Nagatsuta-cho, Midoriku, Yokohama, Kanagawa 226-8502, Japan. ${ }^{2}$ School of Engineering, Tokyo Institute of Technology, 4259-G5-27 Nagatsuta-cho, Midori-ku, Yokohama, Kanagawa 226-8502, Japan. ${ }^{3}$ Department of Oral and Maxillofacial Radiology, Tokyo Medical and Dental University, 1-5-45 Yushima, Bunkyo-ku, Tokyo, Japan. ${ }^{4}$ Department of Radiation Therapeutics and Oncology, Tokyo Medical and Dental University, 1-5-45 Yushima, Bunkyo-ku, Tokyo, Japan. ${ }^{5}$ Department of Oral Radiation Oncology, Tokyo Medical and Dental University, 1-5-45 Yushima, Bunkyo-ku, Tokyo 113-8510, Japan.

\section{Acknowledgements}

Mukau Sato, Yukari Saito, Toshio Takayama and Toru Omata: Tokyo Tech contributes to the development of the device and conducting the experiments. Hiroshi Watanabe, Ryoichi Yoshimura and Masahiko Miura: Tokyo Medical and dental university contributes to the overall system design from the medical point of view. 


\section{Competing interests}

The authors declare that they have no competing interests.

\section{Ethics approval and consent to participate}

We have been approved by ethics committee of Tokyo Institute of Technology to conduct experiment in this paper.

\section{Funding}

No funding.

\section{Publisher's Note}

Springer Nature remains neutral with regard to jurisdictional claims in published maps and institutional affiliations.

Received: 31 March 2017 Accepted: 4 September 2017

Published online: 15 September 2017

\section{References}

1. Shibuya H (2009) Current status and perspectives of brachytherapy for head and neck cancer. Int J Clin Oncol 14(1):2-6

2. Yoshimura RI, Shibuya H, Miura M, Watanabe H, Ayukawa F, Hayashi K, Toda K (2009) Quality of life of oral cancer patients after low-dose-rate interstitial brachytherapy. Int J Radiat Oncol Biol Phys 73(3):772-8

3. Matsumoto S, Takeda M, Shibuya H, Suzuki S (1996) T1 and T2 squamous cell carcinomas of the floor of the mouth: results of brachytherapy mainly using 198Au grains. Int J Radiat Oncol Biol Phys 34(4):833-41
4. Long JA, Hungr N, Baumann M, Descotes JL, Bolla M, Giraud JY, Rambeaud JJ, Troccaz JH (2012) Development of a novel robot for transperineal needle based interventions: focal therapy, brachytherapy and prostate biopsies. J Urol 188(4):1369-1374

5. Torabi M, Gupta R, Walsh CJ (2014) Compact robotically steerable imageguided instrument for multi-adjacent-point(map) targeting. IEEE Trans Robot 30(4):802-815

6. Li G, Su H, Shang W, Tokuda J, Hata N, Tempany CM, Fischer GS. A fully actuated robotic assistant for mri-guided prostate biopsy and brachytherapy. In: Proceedings of SPIE medical imaging 2013: image-guided procedures, robotic interventions, and modeling. vol. 8671, p 867117

7. Galdau B, Plitea N, Vaida C, Covaciu F, Pisla, D. Design and control system of a parallel robot for brachytherapy. In: Proceedings of IEEE international conference on automation, quality and testing, robotics. pp 1-6

8. Green TC, Boucher RP, Belman Y (2005) Method and apparatus for loading radioactive seed into brachytherapy needles. U. S. Patent No. 6846283:B2

9. Yu E, Lewis C, Trejos AL, Patel RV, Malthaner RA (2011) Lung cancer brachytherapy: robotics-assisted minimally invasive approach. J Curr Respir Med Rev 7(5):340-353

10. microSelectron Digitaltreatment Delivery. https://www.elekta.com/ brachytherapy/microselectron-digital-treatment-delivery.html

11. Arakawa T, Takayama T, Omata T, Shibuya H, Miura M, Watanabe H (2011) Development of remote brachytherapy insertion device for oral cancer. J Japan Soc Comput Aided Surg 13:334-335

12. PartsFeeders. https://www.youtube.com/ results?search_query=parts+feeder

13. SEKI Nut and Bolt Feeders. http://www.geschmidt.com/ seki-air-rod-feeder-feedermate-and-bolt-feeder-ge-schmidt/

\section{Submit your manuscript to a SpringerOpen ${ }^{\circ}$ journal and benefit from:}

- Convenient online submission

$\checkmark$ Rigorous peer review

- Open access: articles freely available online

- High visibility within the field

- Retaining the copyright to your article

Submit your next manuscript at $\boldsymbol{\nabla}$ springeropen.com 